\section{Kidney Blood Pressure Research}

\title{
Trace Urine Albumin and Mortality: Kangbuk Samsung Health Study
}

\author{
Eun Na Han ${ }^{a}$ Kyu Beck Lee ${ }^{b}$ Hyang Kim $^{b}$ Young Youl Hyun ${ }^{b}$ \\ aPyunHan Yonsei Medical Clinic, Seoul, 'Division of Nephrology, Department of Internal Medicine, \\ Kangbuk Samsung Hospital, Sungkyunkwan University School of Medicine, Seoul, Republic of Korea
}

\section{Key Words}

Urine dipstick test $\cdot$ Trace albumin $\bullet$ Specific gravity $\bullet$ Mortality

\begin{abstract}
Background/Aims: The importance of a trace albumin on the urine dipstick test is not well known and is frequently disregarded in clinical practice. The aim of this study is to investigate the role of trace albuminuria in predicting all-cause mortality in Korean adults. Methods: In this retrospective cohort study, we analyzed 347,938 Korean adults who underwent urine dipstick test from 2002 to 2012 in a health examination program. The participants were divided into three groups according to dipstick negative, trace $( \pm)$, and overt $(\geq 1+)$ albuminuria. The trace group was further stratified by urine specific gravity $(\mathrm{SG})$ and classified as either high $(\geq 1.020)$ or low $(\leq 1.015)$ SG. Mortality data was obtained from STATICS KOREA, a Korean federal statistical organization. Results: During a median follow-up period of 5.9 years, 1,855 deaths occurred. Mortality was compared to negative group and adjusted for potential risk factors. Mortality did not increase in the trace group (HR, 1.19; 95\% CI, 0.97-1.47; P=0.097), but increased in the overt $(\mathrm{HR}, 1.69 ; 95 \% \mathrm{CI}, 1.38-2.07 ; \mathrm{P}<0.001)$ albuminuria group. When the trace group was subdivided according to urine $S G$, mortality did not increase in the high SG (HR, 1.09; 95\% CI, 0.86-1.37; $\mathrm{P}=0.488)$ group, but increased in the low SG (HR, 1.84; 95\% $\mathrm{CI}, 1.19-2.83 ; \mathrm{P}=0.006)$ and overt $(\mathrm{HR}, 1.70 ; 95 \% \mathrm{CI}, 1.39-2.08 ; \mathrm{P}<0.001)$ groups. Conclusion: Trace albumin with low SG in urine dipstick test was associated with higher all-cause mortality in Korean adults. We should not neglect the significance of trace albumin and SG when interpreting urinalysis results.

(C) 2018 The Author(s)

Published by S. Karger AG, Basel
\end{abstract}

\section{Introduction}

Albuminuria is a well-known risk factor of the progression of renal disease and development of cardiovascular disease and has been shown to be associated with higher

\footnotetext{
Young Youl Hyun, MD, PhD Division of Nephrology, Department of Internal Medicine, Kangbuk Samsung Hospital, Sungkyunkwan University School of Medicine, 29 Saemunan-ro, Jongro-Gu, Seoul 03181 (Republic of Korea) Tel. +82-2-2001-2491, Fax 82-2-2001-2491, E-Mail femur0@naver.com
} 


\section{Kidney Blood Pressure Research}

Han et al.: Trace Albuminuria and Mortality

mortality in subjects with or without chronic kidney disease (CKD). For this reason, the 2012 KDIGO guidelines recommended including albuminuria as a parameter in the staging of CKD [1].

As the sensitivity of albumin testing has improved, questions regarding the clinical importance of low-level albuminuria have also emerged. Moderately increased urinary albumin, also called microalbuminuria, is an early manifestation of diabetic nephropathy, [2] a predictor of cardiovascular diseases and is associated with higher mortality from cardiovascular and all other causes [3]. Furthermore, recent studies have shown that even low-grade albuminuria, i.e., that below the current microalbuminuria threshold, is predictive of cardiovascular disease, progression of chronic kidney disease, and increased mortality [4].

Although quantitative methods of measuring urine albumin, such as the albumin to creatinine ratio (ACR) and the protein to creatinine ratio (PCR), are widely used clinically, the dipstick urinalysis is still a fundamental part of an evaluation for renal disease due to its ease of use and the diverse information it yields at a low cost. This method of measuring albumin is semi-quantitative, with values reported as negative, trace $( \pm), 1+, 2+, 3+$, and $4+$. Trace values are often overlooked in clinical practice, as the clinical significance of this value is not well understood. K-DOQI guidelines recommend quantitative confirmation of proteinuria only in the case of positive $(>1+)$ dipstick results [5].

The aim of this study was to investigate the role of trace albuminuria measured via dipstick urinalysis in all-cause mortality within the Korean adults. We analyzed mortality data from all causes in participants of a health check-up program at a tertiary Korean hospital and categorized the data according to urine albumin level.

\section{Materials and Methods}

\section{Study population}

The Kangbuk Samsung Health Study is a cohort study of South Korean men and women 18 years of age or older who underwent comprehensive annual or biennial health examinations from 2002 to 2012 at Kangbuk Samsung Hospital Health Screening Center in Seoul and Suwon, South Korea [6]. In South Korea, employees are required to participate in annual or biennial health examinations by the Industrial Safety and Health Law. Our participants included employees, their family members, and individuals who underwent voluntary check-ups. Initially, 396,815 participants were included. 44,460 were excluded from the study due to missing data related to key variables. 4,218 subjects were excluded due to a history of cancer. Finally, we excluded 199 subjects who died within one year of the study. This is in order to avoid confounding by death from preexisting diseases. Subsequently, data for the remaining 347,938 participants were analyzed. This study was approved by the Institutional Review Board at Kangbuk Samsung Hospital.

\section{Measurements}

The health check-up included anthropometric measurements, biochemistry studies, and a selfadministered questionnaire on medical history, medication use, and health-related behaviors. Trained staff obtained the anthropometric data, systolic and diastolic blood pressure (BP), and blood samples according to standardized protocols. Body mass index was calculated as weight in kilograms divided by the square of the height in meters. Blood specimens were sampled from the antecubital vein after an at least 10-hour fast.

Serum glucose, total cholesterol, triglycerides, LDL-C, HDL-C, and uric acid levels were determined using an enzymatic colorimetric assay. Serum high-sensitivity C-reactive protein (hsCRP) was determined with a particle-enhanced immunoturbidimetric assay on a Modular Analytics P800 apparatus (Roche Diagnostics, Tokyo, Japan). Serum insulin was measured with an electrochemiluminescence immunoassay on a Modular Analytics E170 apparatus (Roche Diagnostics).

Insulin resistance was assessed with homeostasis model assessment of insulin resistance (HOMA-IR). The HOMA-IR was calculated according to the following equation: fasting blood insulin $(\mu \mathrm{U} / \mathrm{ml}) \times$ fasting blood glucose (mg/dl) / 405. The Department of Laboratory Medicine of the Kangbuk Samsung Hospital 


\section{Kidney Blood Pressure Research}

has been accredited by the Korean Society for Laboratory Medicine and the Korean Association of Quality Assurance for Clinical Laboratories and participates in the College of American Pathologists Proficiency Testing surveys.

During the study period, serum creatinine level was measured using the kinetic alkaline picrate method (Jaffe method) on an automated chemistry analyzer (Advia 1650a Autoanalyzer [Bayer Diagnostics] from 2002 to 2009; Modular D2400 [Roche] from 2010 to 2013). Because the laboratory method used to measure serum creatinine level from 2002 to 2009 was not traceable in isotope dilution mass spectrometry, the estimated glomerular filtration rate (eGFR) was calculated using two different four-variable Modification of Diet in Renal Disease Study equations [7] according to the method of creatinine measurement used.

Urine albumin and specific gravity (SG) were measured semi-quantitatively by dipstick urinalysis (URiSCAN Urine test strips, YD Diagnostics) using fresh, midstream urine samples. Urine albumin was reported as the following 6 values: negative, trace $( \pm$ ), $1+, 2+, 3+$, and $4+$ (corresponding to albumin levels of undetectable or < $10 \mathrm{mg} / \mathrm{dL}, 10$ to $29 \mathrm{mg} / \mathrm{dL}, 30$ to $99 \mathrm{mg} / \mathrm{dL}, 100$ to $299 \mathrm{mg} / \mathrm{dL}, 300$ to $999 \mathrm{mg} / \mathrm{dL}$, and $1000 \mathrm{mg} / \mathrm{dL}$ or greater, respectively). The reported urine SG values ranged from 1.0025 to 1.0350 .

Hypertension was defined as systolic BP $\geq 140 \mathrm{mmHg}$, diastolic $\mathrm{BP} \geq 90 \mathrm{mmHg}$, history of hypertension, or use of an anti-hypertensive medication. Diabetes mellitus was defined as fasting serum glucose $\geq 126$ $\mathrm{mg} / \mathrm{dL}$, a history of diabetes, or use of medication for diabetes. Dyslipidemia was defined as total cholesterol $>240 \mathrm{mg} / \mathrm{dL}$ or a history of dyslipidemia. Cardiovascular disease (CVD) included history of heart disease, coronary disease, and stroke.

\section{Acquisition of mortality data}

Mortality data was gathered on study participants through December 31, 2012, from their baseline examination reports. Survival ascertainment was based on the national death certificate data obtained from the STATICS KOREA, a Korean federal statistical organization. All Koreans deaths are to be reported to the Korea National Statistical Office, and death certificate data for Korean adults are virtually complete.

\section{Statistical analysis}

Participants were divided into three groups according to urine albumin results: negative, trace $( \pm)$, and overt $(\geq 1+)$. Then, the trace group was further stratified by urine SG: high $(\geq 1.020)$ and low $(\leq 1.015)$. Continuous variables were compared between the three groups using an analysis of variance or the KruskalWallis test. Categorical variables were expressed as percentages and compared between groups using the $\chi^{2}$ test. Kaplan-Meier curves were used to display mortality rate in each group. Cox proportional hazards models were used to estimate adjusted hazard ratios (HRs) and 95\% confidence intervals (CIs) for mortality data in participants. The group with negative urine albumin was used as the reference category. Model 1 was adjusted for age and sex. Model 2 was further adjusted for BMI, hypertension, diabetes, dyslipidemia, CVD, and eGFR. Model 3 was further adjusted for current smoking, daily alcohol intake, and number of vigorous exercise sessions per week. We verified the proportional hazards assumption using the Schoenfeld residuals method (estat phtest command in Stata). Subgroup analyses were performed on participants with normal eGFR (eGFR $\geq 60 \mathrm{ml} / \mathrm{min} / 1.73 \mathrm{~m}^{2}$ ) and on participants without hypertension, diabetes, or CVD (i.e., those unlikely to be taking renin-angiotensin system blockers), as well as those $\geq 30$ years of age (who are not likely to have orthostatic proteinuria). Statistical analyses were performed using Stata Version 14.1 (StataCorp LP, College Station, TX, USA).

\section{Results}

The baseline characteristics of study participants classified by urine albumin level are summarized in Table 1. Overall, the overt group had the worst cardiometabolic profile. Participants with overt albuminuria were more likely to be older, male, a current smoker, to drink alcohol, and to suffer from hypertension, diabetes, dyslipidemia, or CVD. The baseline systolic and diastolic BPs, fasting glucose, HOMA-IR, total and LDL cholesterol, uric acid, and hsCRP increased with worsening albuminuria, whereas eGFR decreased with increasing 


\section{Kidney Blood Pressure Research}

Table 1. Characteristics of participants by albuminuria category. Note: Values are reported as number (percentage) for categorical variables and as mean \pm standard deviation or median (interquartile range) for continuous variables. P values were obtained using a t-test or the Mann-Whitney $U$ test for continuous variables and the $\chi 2$ test for categorical variables. Abbreviation: BMI, body mass index; BP, blood pressure; HOMA-IR, homeostasis model assessment of insulin resistance; LDL, low-density lipoprotein; HDL, highdensity lipoprotein; eGFR, estimated glomerular filtration rate; hsCRP, high-sensitivity C-reactive protein; CVD, cardiovascular disease. ${ }^{*}$ Exercised $\geq 3$ times per week

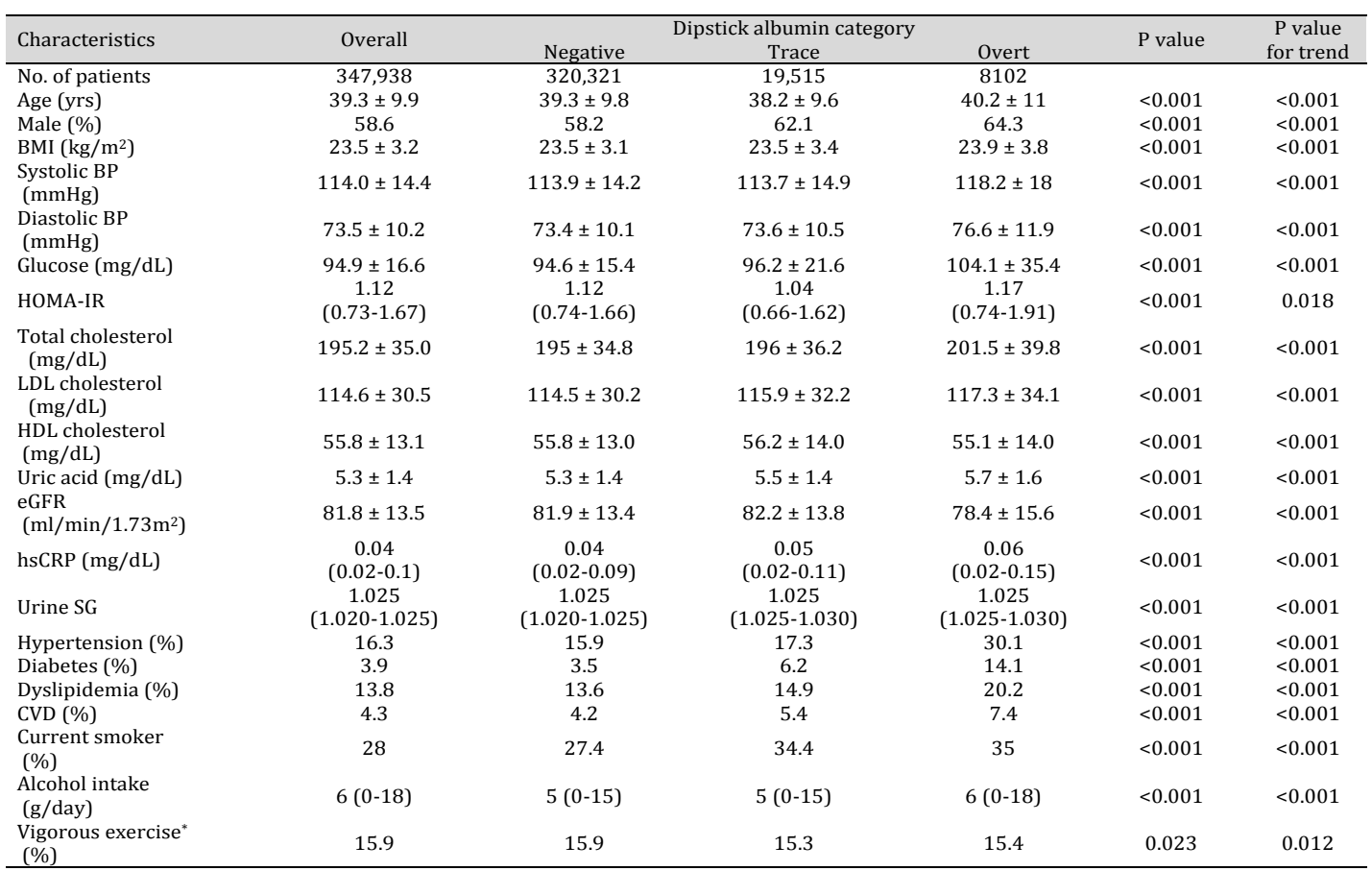

albuminuria. The number of participants with trace albumin was 19,515, and the prevalence rate was $5.6 \%$.

The median length of follow-up for the cohorts was 5.9 years (range 0-10.99, $1,981,939$ person-years). During this period, 1,855 deaths occurred, which corresponds to a mortality rate of 93.6 per 100,000 person-years. Major causes of death were cancer ( $\mathrm{n}=897,48.4 \%)$, suicide and accidents $(\mathrm{n}=369,19.9 \%)$, and cardiovascular disease $(n=256,13.8 \%)$. The survival curves for all-cause mortality by urine albumin are illustrated in Fig. 1. The survival rate was consistently higher in participants without albuminuria over the entire follow-up period.

When the risk of death from all causes was analyzed according to category of urine

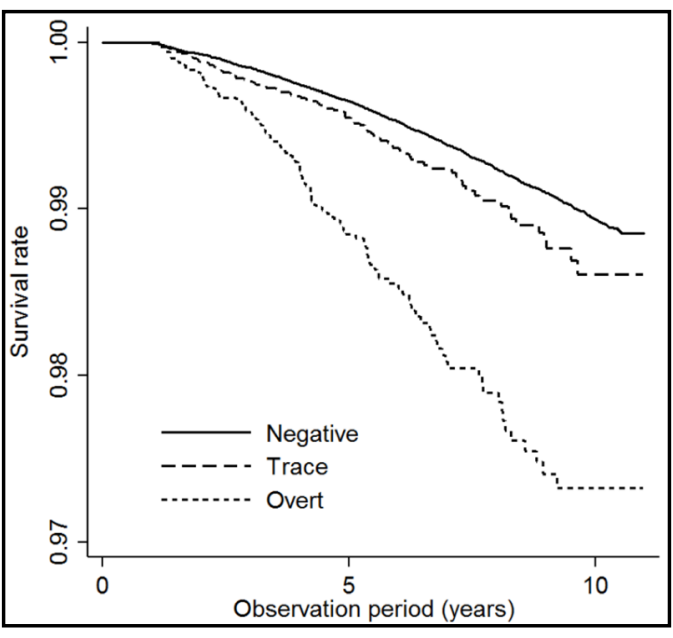

Fig. 1. Survival curves for all-cause mortality according to dipstick urine albumin. albumin concentration, the group with overt albuminuria had a significantly increased HR compared to the negative group in each model (Table 2). The analysis was adjusted for age, sex, body mass index, hypertension, diabetes, dyslipidemia, CVD, eGFR, smoking, alcohol intake, and exercise in the fully adjusted model 3. In this model, HR did not increase in the trace group (HR, 1.19; 95\% CI, 0.94-1.47; $P=0.097$ ), but increased in the overt group (HR, 1.69; 95\% CI, 1.38-2.07; $P<0.001)$. 


\section{Kidney Blood Pressure Research}

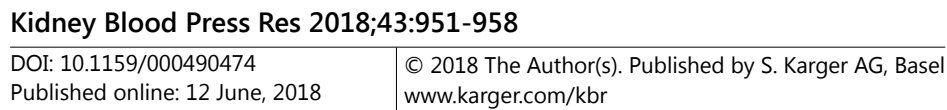

Han et al.: Trace Albuminuria and Mortality

Table 2. Risk of death from all causes according to dipstick albumin category. ${ }^{a}$ Model 1: adjusted for age and sex. ${ }^{b}$ Model 2: adjusted for age, sex, BMI, hypertension, diabetes, dyslipidemia, cardiovascular disease, and eGFR. 'Model 3: adjusted for age, sex, BMI, hypertension, diabetes, dyslipidemia, cardiovascular disease, eGFR, current smoker, alcohol intake, and vigorous exercise frequency. Abbreviations: CI, confidence interval; SG, specific gravity; BMI, body mass index; eGFR, estimated glomerular filtration rate. ${ }^{*}$ per $10^{5}$ person-years

\begin{tabular}{|c|c|c|c|c|c|c|}
\hline \multirow{2}{*}{ Urine dipstick } & \multirow{2}{*}{ Person-years } & \multirow{2}{*}{$\begin{array}{l}\text { Number } \\
\text { of events }\end{array}$} & \multirow{2}{*}{ Mortality rate* } & \multicolumn{3}{|c|}{ Adjusted HR (95\% CI) } \\
\hline & & & & Model $1^{\mathrm{a}}$ & Model $2^{\mathrm{b}}$ & Model 3c \\
\hline \multicolumn{7}{|l|}{ Albumin alone } \\
\hline Negative & 1847845.7 & 1653 & 89.5 & Reference & Reference & Reference \\
\hline Trace & 90095.0 & 95 & 105.4 & $1.35(1.10-1.66)$ & $1.27(1.03-1.56)$ & $1.19(0.97-1.47)$ \\
\hline Overt & 43998.2 & 107 & 243.2 & $2.13(1.75-2.59)$ & $1.82(1.49-2.23)$ & $1.69(1.38-2.07)$ \\
\hline \multicolumn{7}{|l|}{ Albumin \& SG } \\
\hline Negative & 1847845.7 & 1653 & 89.5 & Reference & Reference & Reference \\
\hline Trace/high SG & 79899.8 & 74 & 92.6 & $1.23(0.97-1.55)$ & $1.16(0.92-1.46)$ & $1.09(0.86-1.37)$ \\
\hline Trace/low SG & 10195.2 & 21 & 206.0 & $2.05(1.33-3.16)$ & $1.90(1.23-2.93)$ & $1.84(1.19-2.83)$ \\
\hline Overt & 43998.2 & 107 & 243.2 & $2.13(1.75-2.59)$ & $1.82(1.49-2.23)$ & $1.70(1.39-2.08)$ \\
\hline
\end{tabular}

Table 3. Risk of death from all causes according to dipstick albuminuria category (Kangbuk Samsung Health Study, Seoul, Republic of Korea). adjusted for age, sex, BMI, hypertension, diabetes, dyslipidemia, cardiovascular disease, eGFR, current smoker, alcohol intake, and vigorous exercise frequency. ${ }^{b}$ adjusted for age, sex, BMI, dyslipidemia, eGFR, current smoker, alcohol intake, and vigorous exercise frequency. ${ }^{\text {cadjusted }}$ for age, sex, BMI, hypertension, diabetes, dyslipidemia, cardiovascular disease, eGFR, current smoker, alcohol intake, and vigorous exercise frequency. Abbreviation: $\mathrm{CI}$, confidence interval; SG, specific gravity; CVD, cardiovascular disease. ${ }^{*}$ per $10^{5}$ person-years

\begin{tabular}{|c|c|c|c|c|c|}
\hline Subgroup & Urine dipstick & Person-years & $\begin{array}{l}\text { Number } \\
\text { of events }\end{array}$ & Mortality rate* & adjusted HR $(95 \% \mathrm{CI})$ \\
\hline \multirow{5}{*}{$\begin{array}{l}\mathrm{eGFR} \geq 60 \\
\left(\mathrm{~mL} / \mathrm{min} / 1.73 \mathrm{~m}^{2}\right)^{\mathrm{a}} \\
\mathrm{n}=340,094\end{array}$} & Negative & 1799447.6 & 1487 & 82.6 & Reference \\
\hline & Trace/high SG & 77450.2 & 66 & 85.2 & $1.09(0.85-1.40)$ \\
\hline & Trace/low SG & 9757.2 & 19 & 194.7 & $2.37(1.50-3.73)$ \\
\hline & Overt & 39870.4 & 75 & 188.1 & $1.67(1.32-2.12)$ \\
\hline & Negative & 1453029.9 & 839 & 57.7 & Reference \\
\hline \multirow{4}{*}{$\begin{array}{l}\text { No hypertension, diabetes, or CVD } \\
n=274,305\end{array}$} & Trace/high SG & 58957.2 & 28 & 47.5 & $1.05(0.72-1.53)$ \\
\hline & Trace/low SG & 7354.9 & 8 & 108.8 & $2.29(1.14-4.60)$ \\
\hline & Overt & 25839.2 & 24 & 92.9 & $1.88(1.25-2.82)$ \\
\hline & Negative & 1706791.1 & 1625 & 95.2 & Reference \\
\hline \multirow{3}{*}{$\begin{array}{l}\text { Age } \geq 30 \text { years }^{c} \\
n=315,876\end{array}$} & Trace/high SG & 70723.2 & 73 & 103.2 & $1.10(0.87-1.39)$ \\
\hline & Trace/low SG & 9311.3 & 21 & 225.5 & $1.87(1.21-2.88)$ \\
\hline & Overt & 39458.4 & 106 & 268.6 & $1.70(1.39-2.09)$ \\
\hline
\end{tabular}

In the next analysis, when the trace group was analyzed according to urine SG, trace albuminuria combined with low urine SG significantly increased HR compared to the negative group in each model (Table 2). In model 3, HR did not increase in the trace albumin/high SG group (HR, 1.09; 95\% CI, 0.86-1.37; $P=0.488$ ), but increased in the trace albumin/low SG (HR, 1.84; 95\% CI, 1.19-2.83; $P=0.006)$ and overt (HR, 1.70; 95\% CI, 1.39-2.08; $P<0.001)$ groups.

We also evaluated the combined effect of trace urine dipstick albumin and low urine SG on all-cause mortality in different subgroups. In participants with preserved eGFR ( $\geq 60 / \mathrm{ml} /$ $\min / 1.73 \mathrm{~m}^{2}$ ), HR increased in the trace albumin/low SG (HR, 2.37; 95\% CI; $1.50 \pm 3.73$ ) and overt albuminuria (HR, 1.67; 95\% CI; 1.32 \pm 2.12 ) groups compared to the negative group. This association was similar in the other subgroups (Table 3 ).

\section{Discussion}

In this retrospective study of a large Korean cohort, we showed that trace urinary albumin, as measured by dipstick urinalysis, is an independent risk factor of all-cause mortality. However, the ability of trace albuminuria to predict the risk of death was effective only when interpreted along with urine SG. This association was significant after adjustments for various risk factors related to mortality, and the association persisted even in subgroups unlikely to be taking a renin-angiotensin system blocker (i.e., preserved eGFR, and without 


\section{Kidney Blood Pressure Research}

hypertension, diabetes or CVD) and in participants unlikely to have orthostatic proteinuria (age $\geq 30$ years).

Several studies have highlighted the clinical significance of trace albuminuria. Sam et al. examined 185 samples of urine and found abnormal albumin excretion in $87 \%$ of those with trace albuminuria readings. They concluded that trace proteinuria usually corresponds to microalbuminuria, which is eliminated based on a sample negative for proteinuria [8]. Konta et al. compared dipstick tests with urine ACR in samples obtained from 2, 321 Japanese patients presenting for check-ups. By reclassifying trace proteinuria as a positive result, the sensitivity of the urine protein dipstick test for albuminuria improved from $23.3 \%$ to $37.1 \%$ without specificity reduction; thus, it was concluded that trace proteinuria could potentially be a useful indicator of microalbuminuria [9].

$\mathrm{SG}$ is measured to estimate urine concentration and defined as the weight of a solution relative to that of an equal volume of water [10]. Some studies have used urine SG to compensate for the limitations of the urine dipstick test because it correlates with urine concentration. Marigel et al. analyzed 2,098 samples from a hospital laboratory database and created a model predicting urine PCR from the dipstick protein and urine SG, which showed that trace proteinuria along with low urine SG could be used to detect pathological proteinuria [11]. Natsuko et al. analyzed 582 urine samples obtained from 283 pregnant women to assess the viability of using the dipstick test combined with urine SG as a method to diagnosis clinically significant proteinuria. In the study, the prevalence of pathological proteinuria rose from $0.7 \%$ to $5.0 \%$ when a trace dipstick protein level was detected concurrent with low urine SG [12].

Several studies have shown that dipstick albuminuria predicts higher cardiovascular and all-cause mortality rates. However, most of these studies used both trace and negative in albumin as negative control groups [13-18]. Only two studies have implicated trace dipstick albuminuria as risk factor of higher mortality. Participants of the 15th biennial examination in Framingham Heart Study were followed for 10.5 years. In that study, trace proteinuria was associated with a 30\% increase in all-cause mortality [19]. However, study subjects were mostly white and elderly with a high cardiovascular disease burden. The second study was a collaborative investigation performed by the Chronic Kidney Disease Prognosis Consortium $[20,21]$. In this meta-analysis of general population cohort studies, trace urine albumin was an independent predictor of mortality risk from cardiovascular and all other causes across all eGFR categories. That study also had limitations. The individual studies analyzed did not have uniform protocols, the laboratories used were not centralized or standardized, and some of the studies did not meet the proportional hazard assumption [20].

Our study verified the clinical significance of trace albuminuria as a risk factor of all-cause mortality through a standardized check-up protocol and laboratory methods conducted at a single center. Furthermore, the results were significant even in a relative young and healthy population without comorbidities, which had not been demonstrated in prior studies. The mortality HR of 1.84 found in the trace albumin/low SG group was somewhat higher than the $1.30 \sim 1.44$ reported previously $[19,20]$; this is likely due to the inclusion of urine SG data in the risk prediction.

Currently, most guidelines and authorities do not recommend the urine dipstick test be part of routine screening for the general population due to lack of evidence [22, 23]. However, this test is convenient, does not require high-tech equipment, and can reveal various characteristics of urine, including $\mathrm{pH}, \mathrm{SG}$, glucose, albumin, bilirubin, ketones, blood, leukocyte esterase, and nitrites, at a nominal cost. For example, seven components of urine dipstick tests including microscopy costs only 2.2 US dollars in Korea, lower than the 2.7 US dollars it costs to obtain a urine PCR or 17.4 dollars for urine ACR. For these reasons, dipstick urinalysis is commonly used to detect renal and urologic diseases in the clinic $[24,25]$ and is part of national screening in the general population of some Asian countries [26-28]. This study suggests that the urine dipstick albumin test and urine SG are useful tools in identifying people at high risk of death. To our knowledge, this is the first study to demonstrate that even 


\section{Kidney \\ Blood Pressure Research}

a single measurement of trace albuminuria with low urine SG predicts a higher mortality risk in a relative healthy Asian adult at the single center level.

This study has some limitations. First, this retrospective cohort participated in a health check-up program and was mostly working individuals. There may have been a selection bias; therefore, these participants might not be representative of the general population. However, verifying the association between trace albuminuria and high mortality in this relatively healthy population was an interesting characteristic of this study, as mentioned above. Second, we do not have the data on the specific classes of antihypertensive medications used. The use of certain medications, such as angiotensin-converting-enzyme inhibitors or angiotensin II receptor blockers, may have influenced the results, although the results were unchanged when we excluded participants who might be on these drugs. Despite these limitations, the relatively large sample size, uniform and standardized measurements, and adjustments for various data associated with mortality are all strengths of this study and differentiate it from prior studies.

\section{Conclusion}

In a retrospective cohort of 347,938 Korean adults participating in a health check-up program, the all-cause mortality risk was significantly increased in subjects with dipstick albuminuria. Even trace albuminuria, despite it being frequently overlooked in clinical practice, was associated with a higher mortality risk when combined with low urine SG. These data suggest that the urine dipstick albumin test used in combination with SG may be useful to stratify individuals by mortality risk, and we should not neglect the clinical significance of trace albumin and SG when interpreting the results of urine dipstick test.

\section{Disclosure Statement}

All the authors declared no conflict of interest and no funding source.

\section{References}

1 KDIGO CKD Work Group: KDIGO 2012 Clinical Practice Guideline for the Evaluation and Management of Chronic Kidney Disease. Kidney Int Suppl 2013;3:1-150.

-2 Gross JL, de Azevedo MJ, Silveiro SP, Canani LH, Caramori ML, Zelmanovitz T: Diabetic nephropathy: diagnosis, prevention, and treatment. Diabetes Care 2005;28:164-176.

-3 Weir MR: Microalbuminuria and cardiovascular disease. Clin J Am Soc Nephrol 2007;2:581-590.

4 Kovesdy CP, Lott EH, Lu JL, Malakauskas SM, Ma JZ, Molnar MZ, Kalantar-Zadeh K: Outcomes associated with microalbuminuria: effect modification by chronic kidney disease. J Am Coll Cardiol 2013;61:16261633.

5 National Kidney Foundation: K/DOQI clinical practice guidelines for chronic kidney disease: evaluation, classification, and stratification. Am J Kidney Dis 2002;39:S1-266.

-6 Zhang Y, Chang Y, Ryu S, Cho J, Lee WY, Rhee EJ, Kwon MJ, Pastor-Barriuso R, Rampal S, Han WK, Shin H, Guallar E: Thyroid hormone levels and incident chronic kidney disease in euthyroid individuals: the Kangbuk Samsung Health Study. Int J Epidemiol 2014;43:1624-1632.

$>7$ Levey AS, Coresh J, Greene T, Marsh J, Stevens LA, Kusek JW, Van Lente F: Expressing the Modification of Diet in Renal Disease Study equation for estimating glomerular filtration rate with standardized serum creatinine values. Clin Chem 2007;53:766-772.

-8 Sam R, Shaykh MS, Pegoraro AA, Khalili V, Hristea I, Singh AK, Arruda JA, Dunea G: The significance of trace proteinuria. Am J Nephrol 2003;23:438-441. 


\section{Kidney \\ Blood Pressure Research}

- Konta T, Hao Z, Takasaki S, Abiko H, Ishikawa M, Takahashi T, Ikeda A, Ichikawa K, Kato T, Kawata S, Kubota I: Clinical utility of trace proteinuria for microalbuminuria screening in the general population. Clin Exp Nephrol 2007;11:51-55.

10 Skorecki K, Chertow GM, Marsden PA, Taal MW, Yu ASL: Brenner \& Rector's The Kidney, ed 10th edition., revised. Philadelphia, PA, Elsevier, 2016.

11 Constantiner M, Sehgal AR, Humbert L, Constantiner D, Arce L, Sedor JR, Schelling JR: A dipstick protein and specific gravity algorithm accurately predicts pathological proteinuria. Am J Kidney Dis 2005;45:833-841.

12 Makihara N, Yamasaki M, Morita H, Yamada H: A dipstick test combined with urine specific gravity improved the accuracy of proteinuria determination in pregnancy screening. Kobe J Med Sci 2011;56:E165172.

13 Muntner P, He J, Hamm L, Loria C, Whelton PK: Renal insufficiency and subsequent death resulting from cardiovascular disease in the United States. J Am Soc Nephrol 2002;13:745-753.

14 Wen CP, Cheng TY, Tsai MK, Chang YC, Chan HT, Tsai SP, Chiang PH, Hsu CC, Sung PK, Hsu YH, Wen SF: Allcause mortality attributable to chronic kidney disease: a prospective cohort study based on 462293 adults in Taiwan. Lancet 2008;371:2173-2182.

15 Oh SW, Baek SH, Kim YC, Goo HS, Heo NJ, Na KY, Chae DW, Kim S, Chin HJ: Mild decrease in estimated glomerular filtration rate and proteinuria are associated with all-cause and cardiovascular mortality in the general population. Nephrol Dial Transplant 2012;27:2284-2290.

-16 Nagata M, Ninomiya T, Kiyohara Y, Murakami Y, Irie F, Sairenchi T, Miura K, Okamura T, Ueshima H: Prediction of cardiovascular disease mortality by proteinuria and reduced kidney function: pooled analysis of 39,000 individuals from 7 cohort studies in Japan. Am J Epidemiol 2013;178:1-11.

17 Oh SW, Kim S, Na KY, Kim KW, Chae DW, Chin HJ: Glomerular filtration rate and proteinuria: association with mortality and renal progression in a prospective cohort of a community-based elderly population. PLoS One 2014;9:e94120.

-18 Pesola GR, Argos M, Chen Y, Parvez F, Ahmed A, Hasan R, Rakibuz-Zaman M, Islam T, Eunus M, Sarwar G, Chinchilli VM, Neugut AI, Ahsan H: Dipstick proteinuria as a predictor of all-cause and cardiovascular disease mortality in Bangladesh: A prospective cohort study. Prev Med 2015;78:72-77.

19 Culleton BF, Larson MG, Parfrey PS, Kannel WB, Levy D: Proteinuria as a risk factor for cardiovascular disease and mortality in older people: a prospective study. Am J Med 2000;109:1-8.

20 Matsushita K, van der Velde M, Astor BC, Woodward M, Levey AS, de Jong PE, Coresh J, Gansevoort RT: Association of estimated glomerular filtration rate and albuminuria with all-cause and cardiovascular mortality in general population cohorts: a collaborative meta-analysis. Lancet 2010;375:2073-2081.

-21 van der Velde M, Matsushita K, Coresh J, Astor BC, Woodward M, Levey A, de Jong P, Gansevoort RT, van der Velde M, Matsushita K, Coresh J, Astor BC, Woodward M, Levey AS, de Jong PE, Gansevoort RT, Levey A, ElNahas M, Eckardt KU, Kasiske BL, et al.: Lower estimated glomerular filtration rate and higher albuminuria are associated with all-cause and cardiovascular mortality. A collaborative meta-analysis of high-risk population cohorts. Kidney Int 2011;79:1341-1352.

-22 Krogsboll LT: Guidelines for screening with urinary dipsticks differ substantially--a systematic review. Dan Med J 2014;61:A4781.

-23 Krogsboll LT, Jorgensen KJ, Gotzsche PC: Screening with urinary dipsticks for reducing morbidity and mortality. Cochrane Database Syst Rev 2015;1:Cd010007.

-24 Prochazka AV, Lundahl K, Pearson W, Oboler SK, Anderson RJ: Support of evidence-based guidelines for the annual physical examination: a survey of primary care providers. Arch Intern Med 2005;165:1347-1352.

25 Merenstein D, Daumit GL, Powe NR: Use and costs of nonrecommended tests during routine preventive health exams. Am J Prev Med 2006;30:521-527.

26 Lin CY, Sheng CC, Lin CC, Chen CH, Chou P: Mass urinary screening and follow-up for school children in Taiwan Province. Acta Paediatr Taiwan 2001;42:134-140.

27 Imai E, Yamagata K, Iseki K, Iso H, Horio M, Mkino H, Hishida A, Matsuo S: Kidney disease screening program in Japan: history, outcome, and perspectives. Clin J Am Soc Nephrol 2007;2:1360-1366.

28 Kang YU, Kim HY, Choi JS, Kim CS, Bae EH, Ma SK, Kim SW: Metabolic syndrome and chronic kidney disease in an adult Korean population: results from the Korean National Health Screening. PLoS One 2014;9:e93795. 